\title{
The Shielding Effectiveness of a Magnetic Fluid in Radio Frequency Range
}

\author{
B. Dolník ${ }^{a, *}$, M. RajñáK ${ }^{a, b}$, R. Cimbala ${ }^{a}$, I. Kolcunováa ${ }^{a}$, J. KurimskÝ $^{a}$, J. DŽmurA ${ }^{a}$, \\ J. Petrás̆ $\check{S}^{a}$, J. ZBojovskÝ ${ }^{a}$, M. Kosterec ${ }^{a}$, P. KopČAnskÝ ${ }^{b}$, And M. Timko ${ }^{b}$ \\ ${ }^{a}$ Department of Electrical Power Engineering, TU of Košice, Mäsiarska 74, 04120 Košice, Slovakia \\ ${ }^{b}$ Institute of Experimental Physics SAS, Watsonova 47, 04001 Košice, Slovakia
}

\begin{abstract}
This article builds on the previous work and describes the interaction of transformer oil-based magnetic fluid (MF) with the radio frequency (RF) magnetic near-field. Three prepared samples of the MF used as a barrier to magnetic near-field, consist of transformer oil and dispersed magnetite nanoparticles coated with oleic acid. We pay attention to the important area related to the electromagnetic field shielding by the MF. Such sample of the MF may be a good candidate for applications where it is necessary to simultaneously electrically isolate, remove the excess of heat and to shield electromagnetic interference (EMI). We present a method for the determination of shielding effectiveness (SE) of the MF under RF excitation conditions ranging from $500 \mathrm{MHz}$ to $3 \mathrm{GHz}$. We report the effect of magnetic volume fraction in the MF and the effect of the sample thickness on the SE.
\end{abstract}

DOI: 10.12693/APhysPolA.133.585

PACS/topics: 41.20.Jb, 41.20.Gz, 75.50.Mm, 81.70.Ex, 07.50.Hp

\section{Introduction}

Nowadays, with improving technology, global demand for a reliable and quality equipment is greater than ever before. Extended use of electronic devices, closely related to the increased number of communication instruments, digital systems and fast processors, results in the generation of radio frequency interference (RFI). All electronics emit a certain amount of electromagnetic (EM) energy that interacts with other electronics around. If disruptive EM energy causes a malfunction of the electronics (device), it is considered to be interference, or so called electromagnetic interference (EMI) [1-6].

Similarly, people meets (interacts) with EM fields every day. These can be naturally occurring fields in nature and man-made fields when working on technical devices. Extremely high exposure can occur in some of our occupations as well as in our personal activities [7,8]. Electromagnetic shielding is a way to limit the levels of EM fields emitted from sources or to protect people or electrical and electronic devices from external EM fields. In some cases, it is even necessary to completely eliminate radiation from devices, because the lives of many people may depend on the operation of special devices.

The material shielding effectiveness (SE) depends on its geometrical and electrical parameters and on the characteristics of the unperturbed EM field. The most important parameters that affect shield performance are the geometrical configuration, the shielding thickness, and the electrical parameters of the material from which the shield is made [1, 9-11].

\footnotetext{
*corresponding author; e-mail: bystrik.dolnik@tuke.sk
}

\section{Theoretical background}

Measuring EMI SE data in a broad frequency range for newly developed materials is crucial to determine their properties and potential applications. Some of the common techniques for the determination of the SE include $[12,13]$ : open field test, shielded room test, coaxial transmission line test, shielded box test.

In some experimental techniques the dielectric permittivity and magnetic permeability are firstly measured in order to determine the reflection coefficient $R$ and the transmission coefficient $T$ [14-17]. The common definitions of the SE at an arbitrary point $\mathrm{P}$ within the shielded domain are given by

$$
\mathrm{SE}=10 \log \left(\frac{P_{u}}{P_{s}}\right)=20 \log \left(\frac{E_{u}}{E_{s}}\right)=20 \log \left(\frac{H_{u}}{H_{s}}\right) .
$$

In the (1) the numerators represent the amplitudes of the time-harmonic power, electric and magnetic field intensities, measured at $\mathrm{P}$ in the absence of the shield, while the denominators contain their values in the shielded case at the same locations. In the usual measurement situation when the meter readings are given in the volts, more convenient form for (1) is as follows:

$$
\mathrm{SE}_{0[\mathrm{~dB}]}=V_{u[\mathrm{~dB}]}-V_{s[\mathrm{~dB}]} .
$$

The SE can be calculated by measuring of the magnetic field. The SE consists of three components: SE due to reflection $R$, absorption $A$ and multi-reflection $M$

$$
\mathrm{SE}_{[\mathrm{dB}]}=R_{[\mathrm{dB}]}+A_{[\mathrm{dB}]}+M_{[\mathrm{dB}]} \text {. }
$$

It is necessary to consider two radiation zones, when discussing SE: the near field and the far field zones. If the distance from the source of EMI to shielding is less than $\frac{1}{6}$ of the free path wavelength of the EMI to be shielded, the radiation is described as a near field zone. 


\section{Materials and methods}

The investigated magnetic fluid (MF) consists of the $\mathrm{Fe}_{3} \mathrm{O}_{4}$ nanoparticles synthesized from aqueous solution of $\mathrm{Fe}^{2+}$ and $\mathrm{Fe}^{3+}$ ions in the presence of $\mathrm{NH}_{4} \mathrm{OH}$ at $(80-82)^{\circ} \mathrm{C}$ using the chemical co-precipitation method, dispersed in the inhibited insulating mineral oil MOGUL TRAFO CZ-A, PARAMO. The saturation magnetization of the originally prepared ferrofluid sample obtained by a superconducting quantum interference device magnetometer is $23.15 \mathrm{~A} \mathrm{~m}^{2} \mathrm{~kg}^{-1}$, the estimated dc magnetic susceptibility is 0.75 and the average hydrodynamic particle diameter obtained from the dynamic light scattering measurements is $33.28 \mathrm{~nm}$. For the experimental exploration we prepared three samples of the MF with volume fraction $\left(\Phi_{V}\right)$ equal to $0.5 \%, 1.5 \%$ and $6.6 \%$.

The response of the MF to the radio frequency EM field was investigated with two calibrated broadband $(30 \mathrm{MHz}$ to $1 \mathrm{GHz}$ ) magnetic antennas (Agilent 11940A close-field probe). The antennas were arranged in one line at a distance of $3.3 \mathrm{~mm}$ and mutually oriented towards each other, as shown in Fig. 1. The first antenna represents the transmitter (TX), the second antenna represents the receiver (RX). The mutual distance of the antennas is much smaller than the limit of the near field zone at frequency $f=3 \mathrm{GHz}, \lambda_{(3 \mathrm{GHz})}=c / f=10 \mathrm{~cm}$. The manufacturer declares that the antenna works well above $1 \mathrm{GHz}$.

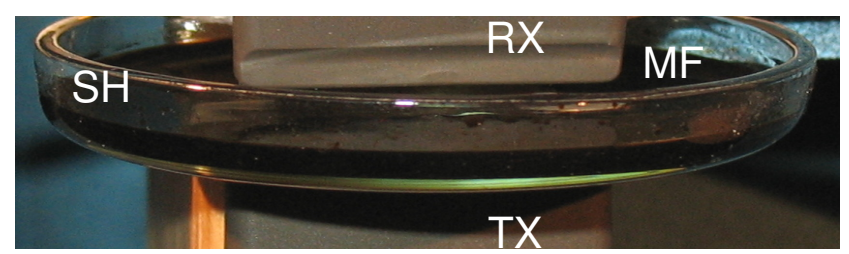

Fig. 1. The measured MF in the sample holder ( $\mathrm{SH})$.

All measurements were performed in the near field zone with predominant magnetic field component, at room temperature $(23 \pm 1){ }^{\circ} \mathrm{C}$ and at a relative air humidity $(34 \pm 3) \%$. The transmitting antenna was supplied with a source of harmonic voltage Agilent N5183A. The received signal level was measured by using the spectrum analyzer R\&S FSH8.

\section{Results and discussion}

As mentioned above, we measured the signal level at the terminal of the receiving antenna in $\mathrm{dB} \mu \mathrm{V}$ at various concentrations and thicknesses of the MF sample. By using the (2) the frequency dependence of the SE in the range from $500 \mathrm{MHz}$ to $3 \mathrm{GHz}$ was calculated. In Fig. 2 the calculated SE in the frequency range from $500 \mathrm{MHz}$ to $1 \mathrm{GHz}$ for the MF with $\Phi_{V}$ of $0.5 \%$ is shown. From the Fig. 2 it can be seen that the SE is approximately linearly dependent on the thickness of the measured sample. In the frequency range from $500 \mathrm{MHz}$ to $1 \mathrm{GHz}$, the

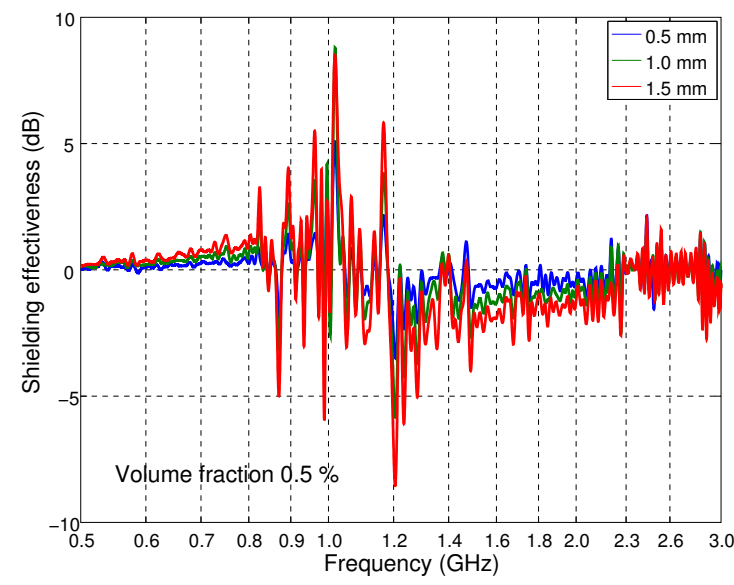

Fig. 2. The SE of the MF with $0.5 \%$ volume fraction.

SE has an increasing trend, with SE increasing from approximately $0 \mathrm{~dB}$ to $8.8 \mathrm{~dB}$. Then, in the frequency range from $1 \mathrm{GHz}$ to $1.2 \mathrm{GHz}$, the $\mathrm{SE}$ has a downward trend through negative values up to $-8.5 \mathrm{~dB}$. In the frequency range from $1.2 \mathrm{GHz}$ to $3 \mathrm{GHz}$, the $\mathrm{SE}$ has an increasing trend, with a $0 \mathrm{~dB}$ SE value reaching approximately at $2.3 \mathrm{GHz}$. The calculated SE for the MF with $\Phi_{V}$ of $1.5 \%$ is shown in Fig. 3. From the Fig. 3 it can be seen that the sample with $\Phi_{V}$ of $1.5 \%$ has a similar trend of the SE across the entire frequency band examined. The calculated SE of the last sample with $\Phi_{V}$ of $6.6 \%$ is shown in Fig. 4. Again, the trend of SE is similar to the previous two samples.

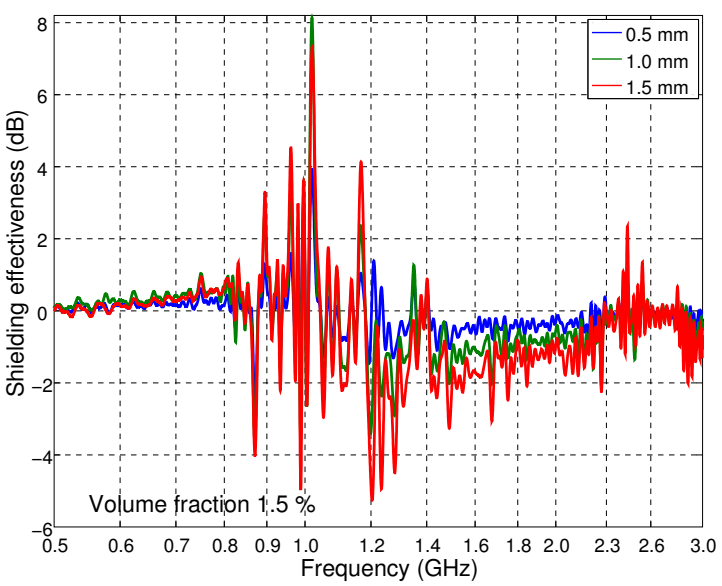

Fig. 3. The SE of the MF with $1.5 \%$ volume fraction.

A negative value of $\mathrm{SE}$ indicates that the magnetic component of the EM field "penetrates" through the shield. A negative value of SE indicates that the magnetic component of the EM field is transmitted through the shield, having an "amplified" amplitude just behind the shield. This phenomenon may be due to multiple reflection component $M_{[\mathrm{dB}]}$ defined in (3). Comparing the SE for the other concentrations of the MF $(0.5 \%$ and 


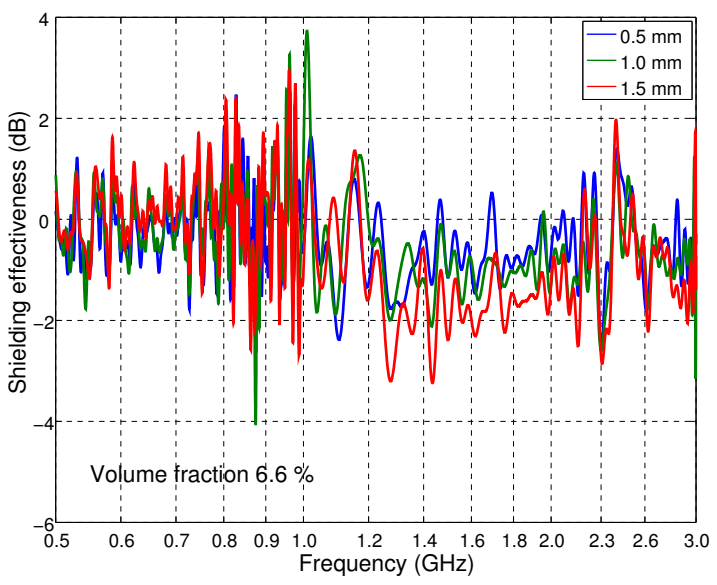

Fig. 4. The SE of the MF with $6.6 \%$ volume fraction.

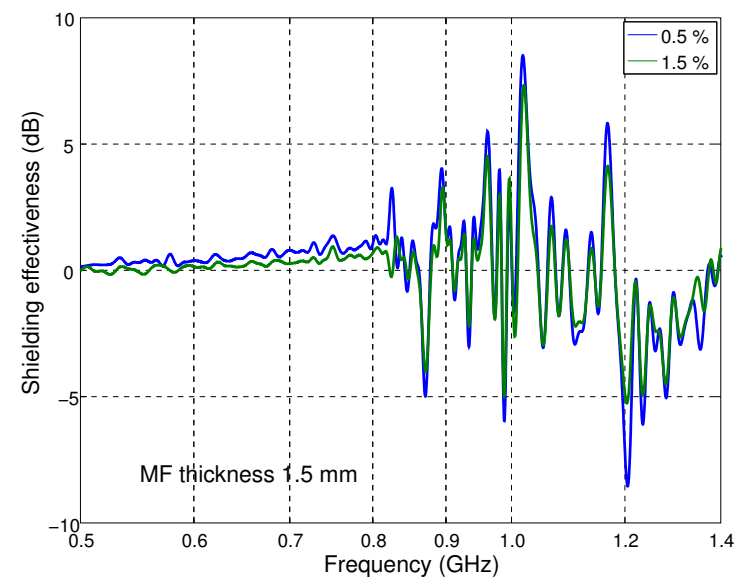

Fig. 5. The SE of the MF with thickness of $1.5 \mathrm{~mm}$ and $0.5 \%$ and $1.5 \%$ volume fraction.

$6.6 \%)$ it was found, that in the frequency range from $500 \mathrm{MHz}$ to $3 \mathrm{GHz}$ the SE decreases with increasing concentration. Figure 5 shows the comparison of the SE on samples with thickness of $1.5 \mathrm{~mm}$ and $\Phi_{V}$ of $0.5 \%$ and in the frequency range from $500 \mathrm{MHz}$ to $1.4 \mathrm{GHz}$.

\section{Conclusions}

Experimental measurements of the shielding effectiveness of the MF based on transformer oil with the high frequency alternating magnetic field were realized. All measurements were realized in the near field zone. The results obtained by measuring, and calculating of the shielding effectiveness pointed to almost linear dependence on the thickness of the sample in the frequency range from $500 \mathrm{MHz}$ to $3 \mathrm{GHz}$. Moreover, it was found that the shielding effectiveness of the magnetic fluid decreases with increasing concentration. Negative shielding effectiveness has coherent frequency window from $1.48 \mathrm{GHz}$ to $2.2 \mathrm{GHz}$. We suppose that this phenomenon is caused by multiple reflection component $M_{[\mathrm{dB}]}$. In the future, we will continue to experiment at higher frequencies.

\section{Acknowledgments}

This work was supported by Slovak Academy of Sciences and Ministry of Education: APVV-15-0438, VEGA 1/0311/15, VEGA 2/0141/16, VEGA 1/0132/15, Ministry of Education Agency for structural funds of EU Project No. 26220220061.

\section{References}

[1] X.C. Tong, Advanced Materials and Design for Electromagnetic Interference Shielding CRC Press, London 2009.

[2] V. Rubežiene, J. Baltušnikaite, S. VarnaiteŽuravliova, A. Sankauskaite, A. Abraitiene, J. Matuzas, J. Electrostat. 75, (2015).

[3] S. Kuester, G.M.O. Barra, J.C. Ferreira Jr., B.G. Soares, N.R. Demarquette, Eur. Polym. J. 77, (2016).

[4] T. Wessapan, P. Rattanadecho, Int. J. Heat Mass Tran. 97, (2016).

[5] D.D.L. Chun, Carbon. 50, (2012).

[6] J. Chen, D. Zhao, H. Ge, J. Wang, Constr. Build. Mater. 84, 66 (2015).

[7] J.C. Lin, Electromagnetic Fields in Biological Systems CRC Press, London 2012.

[8] F.S. Barnes, B. Greenebaum, Bioengineering and Biophysical Aspects of Electromagnetic Fields CRC Press, London 2006.

[9] L. Pengcheng, D. Donghe, G. Lin, G. Yongxin, O. Jianyong, J. Mater. Chem. C. 4, (2016).

[10] H. Zhao, L. Hou, Y. Lu, Mater. Design. 95, (2016).

[11] P.J. Bora, K.J. Vinoy, P.C. Ramamurthy, G. Madras, Materials Research Express, 4, (2017).

[12] J. Hallon, K. Kováč, Procedia Engineering. 69 (2014).

[13] G. Betta, D. Capriglione, C.F.M. Carobbi, M.D. Migliore, Comput. Stand. Inter. $\mathbf{3 3}, 2$ (2011).

[14] P.C. Fannin, I. Malaescu, C.N. Marin, N. Stefu, Eur. Phys. J. E. 29, (2009).

[15] P.C. Fannin, I. Malaescu, N. Stefu, P. Vlăzan, S. Novaconi, P. Sfirloaga, S. Popescu, C. Couper, Mater. Design. 32, (2011).

[16] P. C. Fannin, C.N. Marin, I. Malaescu, N. Stefu, P. Vlăzan, S. Novaconi, S. Popescu, J. Nanopart. Res. 13, (2011).

[17] R. Kumar, S. K. Dhawan, H. K. Singh, A. Kaur, Mat. Chem. Phys. 180, (2016). 